\title{
LOCALLY NILPOTENT SKEW LINEAR GROUPS
}

\author{
by B. A. F. WEHRFRITZ \\ (Received 28th February 1985)
}

\section{Introduction}

Throughout this paper $D$ denotes a division ring with centre $F$ and $n$ a positive integer. A subgroup $G$ of $G L(n, D)$ is absolutely irreducible if the $F$-subalgebra $F[G]$ generated by $G$ is the full matrix ring $D^{n \times n}$. It is completely reducible (resp. irreducible) if row $n$-space $D^{n}$ over $D$ is completely reducible (resp. irreducible), as $D-G$ bimodule in the obvious way. Absolutely irreducible skew linear groups have a more restricted structure than irreducible skew linear groups, see for example [7], [8], [9] and [10]. Here we make a start on elucidating the structure of locally nilpotent such groups.

1.1 Theorem. Let $H$ be a locally nilpotent normal subgroup of the absolutely irreducible skew linear group $G$. If $H$ is either hypercentral or poly abelian-by-periodic then $G / C_{G}(H)$ is periodic. In particular $H$ is centre by locally-finite.

1.2. Theorem. Let $H$ be a locally nilpotent normal subgroup of the completely reducible subgroup $G$ of $G L(n, D)$ and let $S$ be a right Noetherian subring of $D^{n \times n}$ normalized by $G$. Suppose that either $G$ is irreducible or $S=F$, the centre of $D$. Then for every subgroup $K$ of $H$ the subring $S[K]$ of $D^{n \times n}$ generated by $S$ and $K$ is semiprime and right Goldie.

Note that although any subring of a division ring is semiprime with the maximal condition on annihilators it need not be right Goldie; it could be a free ring for example.

Let $G$ be an absolutely irreducible subgroup of $G L(n, D)$ and $H$ a locally nilpotent normal subgroup of $G$. In Section 5 below we analyze in some detail the structure of $G$ and $H$. In view of 1.1 we speculate of course that $H$ is always centre by locally-finite. Our analysis is sufficient to read off immediately that it would suffice to prove this speculation in the special case where

$$
\left(^{*}\right)\left\{\begin{array}{l}
n=1, \text { so } G \text { is a subgroup of the multiplicative group } D^{*} \text { of } D ; \\
D=F[G] \text { is the ring of quotients of } F[H] ; \\
\bar{H}=H /(H \cap F) \text { is torsion-free and } F[H] \text { is crossed product of } F \text { by } \bar{H}
\end{array}\right.
$$

Crossed products are defined in Section 2. Section 5 is the main part of this paper. It makes use of both 1.1 and 1.2. 
The following is an easy consequence of 1.1; it also can be derived from the results of [9].

1.3. A hyperabelian absolutely irreducible skew linear group is abelian by locally-finite.

In fact 3.8 below is slightly stronger than 1.3. The following is of a similar nature. Its proof is in Section 6.

1.4. Let $G$ be an absolutely irreducible skew linear group and let $H$ be a normal subgroup of $G$ with an ascending series whose factors are locally nilpotent-or-finite (e.g. if $H$ is radical). Suppose that the Hirsch-Plotkin radical of $H$ is centre by periodic. Then $H$ and $G / C_{G}(H)$ are both abelian by periodic.

Of course our speculation above asserts that in 1.4 the hypothesis on the HirschPlotkin radical is redundant. The examples in [9] already show that there are no obvious ways of strengthening the conclusions of 1.3 and 1.4. Also if $G$ is a torsion-free locally nilpotent group then $G$ is isomorphic to a (necessarily irreducible) subgroup of the multiplicative group of a division ring (e.g. [5], 13.2.11). This indicates that there is not much scope in weakening the absolute irreducibility in 1.1. Easy examples show that if $G$ is reducible and $S \neq F$ in 1.2 than the conclusion of 1.2 can be false.

Suppose $G$ and $H$ are as in 1.1. Then much information about $H$ can be read off from the results of [11]. For let $Z$ be the centre of $H$. Then $F[Z]$ is reduced by 1.2 and its ring $Q$ of quotients is a direct sum of a finite number of fields. Also by 4.4 below $Q$ is naturally embedded in $F[G]$ and $Q[H]$ by 1.1 is a locally finite-dimensional $Q$-algebra. If $x$ is a regular element of $Q[H]$ then $x \in Q[X]$ for some finitely generated subgroup $X$ of $H$. Then $Q[X]$ is semisimple Artinian by 1.2 and thus $x$ is a unit of $Q[X] \subseteq Q[H]$. Since $Q[H]$ is semiprime and Goldie by 1.2 it folows that $Q[H]$ is a semisimple Artinian locally finite-dimensional $Q$-algebra. This is the situation considered in [11]. For example it follows that

1.5. $H$ contains normal subgroups $A \leqq K$ with $H / K$ finite, $K / A$ torsion-free abelian and $A$ periodic abelian with finite rank at most $n$ and no non-trivial elements of order char $F$.

All rings in this paper, except when the term nilring is used, have an identity, and ring homomorphisms preserve identities. If $X$ is a subset of a ring $R$ then $l_{R}(X)$ denotes the left annihilator and $r_{R}(X)$ the right annihilator of $X$ in $R$. We use P. Hall's calculus of group classes as given in [6], except that we denote the quotient operator by $Q$ and not $H$. For example the group $H$ of 1.4 lies in the class $P L(\mathfrak{N} \cup \mathfrak{F})$.

\section{Ring theoretical lemmas}

2.1. Let $G$ be an irreducible subgroup of $G L(n, D)$ and let $S$ be a subring of $D^{n \times n}$ normalized by $G$. Then $S$ is semiprime.

Proof. Let $n$ be the lower nilradical of $S$ and assume that $n \neq\{0\}$. Note that $n$ is nilpotent ([1] 1.35) and normalized by $G$. Suppose $n^{r}>n^{r+1}=\{0\}$. Then with $V=D^{n}$ regarded as a $D-G$ bimodule in the usual way, $V \mathrm{nt}^{r}$ is a non-zero $D-G$ submodule of $V$, 
which is irreducible. Thus $V \mathfrak{n}^{r}=V$ and so $V \mathfrak{n}=\{0\}$. This implies that $\mathfrak{n}=\{0\}$, a contradiction that completes the proof.

In 2.1 we have only proved what we need. In fact much more is true. If $G$ is an irreducible subgroup of $G L(n, D)$ then $F[G]$ is always prime. If $G$ is completely reducible then $F[N]$ is semiprime for every subnormal subgroup $N$ of $G$. In 2.1 easy examples show that $S$ need not be prime and that if $G$ is only completely reducible then $S$ need not be semiprime.

Say that an absolutely irreducible subgroup $G$ of $G L(n, D)$ is persistent (short for persistently of simple Artinian type) if the $F$-subalgebra $F[H]$ of $D^{n \times n}$ generated by $H$ is simple Artinian for every subnormal subgroup $H$ of finite index. Say that $G$ is prime if $F[N]$ is a prime ring for every normal subgroup $N$ of $G$.

2.2. Let $G$ be an absolutely irreducible subgroup of $G L(n, D)$. Then $G$ has a normal subgroup $H$ of finite index such that $H$ is a subdirect product of groups $H_{i}$, where for each $i, H_{i}$ is a persistent subgroup of $G L\left(n_{i}, D_{i}\right)$ for some integers $n_{i}$ satisfying $\sum n_{i} \leqq n$ and division $F$-algebras $D_{i}$.

Proof. Pick a subnormal subgroup $H_{0}$ of $G$ of finite index such that the composition length of row $n$-space $V=D^{n}$ as $D-H_{0}$ bimodule is maximal and set $H=\bigcap_{g \in G} H_{0}^{g}$. By Clifford's theorem $V$ is completely reducible as $D-H_{0}$ bimodule, so let $V=\oplus V_{i}$ where each $V_{i}$ is $D-H_{0}$ irreducible. Let $\pi_{i}: F\left[H_{0}\right] \rightarrow \operatorname{End}_{D} V_{i}$ be the restriction map and set $H_{i}=H \pi_{i}$.

Let $K$ be any subnormal subgroup of $H_{i}$ of finite index. By the choice of $H_{0}$ we have that $V_{i}$ is $D-K$ irreducible. In particular $V_{i}$ is $D-H_{i}$ irreducible. But $F[H]$ is Artinian by [2] Point 4 and semiprime by 2.1. Thus $F[H]$ and hence also $F\left[H_{i}\right]=F[H] \pi_{i}$ is semisimple. Let $U_{i}$ be an irreducible $F\left[H_{i}\right]$ submodule of $V_{i}$. Then $V_{i}=D U_{i}$ and $d U_{i \simeq F\left[H_{i}\right]} U_{i}$ for every $d \in D^{*}$. Therefore $F\left[H_{i}\right]$ is simple Artinian and hence is a matrix ring of degree $n_{i}$ say over some division $F$-algebra $D_{i}$. Thus $H_{i}$ is an absolutely irreducible subgroup of $G L\left(n_{i}, D_{i}\right)$ in the obvious way. Also $n_{i} \leqq \operatorname{dim}_{D} V_{i}$, so $\sum n_{i} \leqq n$.

Let $F_{i}$ denote the centre of $D_{i}$. We have to prove that $F_{i}[K]$ is simple Artinian. By [2] Point 4 and 2.1 again $F_{i}[K]$ is semisimple Artinian. If $U_{i}$ is now an irreducible $F_{i}[K]$-submodule of $V_{i}$ then again $V_{i}=D U_{i}$ and $d U_{i} \simeq U_{i}$ for $d \in D^{*}$. Therefore $F_{i}[K]$ is simple Artinian.

2.3. Let $G$ be an absolutely irreducible subgroup of $G L(n, D)$. Then the following are equivalent

(a) $G$ is prime

(b) $F[H]$ is prime for every normal subgroup $H$ of $G$ of finite index

(c) $F[H]$ is simple Artinian for all $H$ as in $(b)$.

Proof. Trivially (a) implies (b). For every normal subgroup $H$ of $G$ of finite index $F[H]$ is semisimple Artinian by [2] and 2.1. Thus (b) implies (c). Assume (c) holds and let $N$ be any normal subgroup of $G$. Then $F[N]$ is semiprime by 2.1 and satisfies the 
maximal condition on right annihilators (since $F[G]$ does). Hence $F[N]$ has only a finite number of minimal prime ideals $\mathfrak{p}_{1}, \ldots, \mathfrak{p}_{r}$ by [1] 1.16 and each $\mathfrak{p}_{i}$ is an annihilator ideal. Clearly $G$ permutes the $\mathfrak{p}_{i}$, so $H=\bigcap_{g \in G} N_{G}\left(\mathfrak{p}_{1}\right)^{g}$ is a normal subgroup of $G$ of finite index. By hypothesis $F[H]$ is simple, and $p_{1} H$ is an ideal of $F[H]$. If $p_{1} H=F[H]$ then $l_{F[N]}\left(\mathfrak{p}_{1}\right)=\{0\}$ and $\mathfrak{p}_{1}=r_{F[N]} l_{F[N]}\left(\mathfrak{p}_{1}\right)=F[N]$. This is false and therefore $\mathfrak{p}_{1}=\{0\}$. Thus (a) holds.

2.4. Corollary. If $G$ is a persistent subgroup of $G L(n, D)$ then $G$ is prime.

This follows at once from 2.3. Apart from being useful in the context here 2.2 and 2.4 give a much better approach to 4.3.1 of [7] and to 3.2 and 3.3 of [9]. An obvious question in view of 2.4 is whether a prime absolutely irreducible subgroup of $G L(n, D)$ is always persistent. Presumably the answer is no.

We conclude this section with two conditions for a ring to be a crossed product. If $S$ is a subring of a ring $R$ and if $G$ is a subgroup of the units of $R$ normalizing $S$ such that $R=S[G], S \cap G$ is a subgroup of $G$ and $R$ is a free right $S$-module on a transversal of $S \cap G$ to $G$, then we say that $R$ is a crossed product of $S$ by $G /(S \cap G)$.

2.5. Let $R=F[G]$ be an $F$-algebra, where $F$ is a field and $G$ is a locally nilpotent group of units of $R$, such that for every finite subset $X$ of $R$ there is a finitely generated subgroup $Y$ of $G$ with $F[Y]$ prime and containing $X$. Let $T$ be the torsion subgroup of $G$ and $Z / T$ the centre of $G / T$. Then $R$ is a crossed product of $F[Z]$ by $G / Z$.

Proof. Let $\sum_{i=1}^{r} t_{i} \alpha_{i}=0$ where the $t_{i}$ are distinct elements of a transversal of $Z$ to $G$ and the $\alpha_{i}$ are non-zero elements of $F[Z]$. If $i \neq j$ then $t_{i}^{-1} t_{j} \notin Z$ and so there is some $g_{i j} \in G$ with $t_{i j}=\left[t_{i}^{-1} t_{j}, g_{i j}\right]$ of infinite order. There exists a finitely generated subgroup $Y$ of $G$ with $F[Y]$ prime such that $F[Y]$ contains all the $t_{i}, t_{i}^{-1}$ and $g_{i j}$ and $F[Y \cap Z]$ contains the $\alpha_{i}$.

By a theorem of Zalesskii ([5] 11.4.5) $F[Y]$ is a crossed product of $F[\Delta(Y)]$ by $Y / \Delta(Y)$, where $\Delta(Y)=\left\{y \in Y:\left|y^{Y}\right|<\infty\right\}$. Clearly $T \cap Y$ is finite and $Y \cap Z \leqq \Delta(Y)$. Thus $t_{i}^{-1} t_{j} \in \Delta(Y)$ for some $i \neq j$. Since $Y$ is finitely generated nilpotent, $\Delta(Y)$ is finite by central-in- $Y$. Also $g_{i j} \in Y$. Thus $t_{i j}$ has finite order, a contradiction that completes the proof.

2.6. Let $R$ be a ring, $E$ a division subring of $R$ and $G$ a group of units of $R$ normalizing $E$ such that $E^{*} \leqq G$ and $R=E[G]$. Set $C=C_{R}(E)$. Then $R$ is a crossed product of $E[C]$ over $G / E^{*}(C \cap G)$.

Proof. Let $T$ be a transversal of $E^{*}(C \cap G)$ to $G$ and let $B$ be a basis of $C$ over the centre $F$ of $E$. Clearly $E[C]=\sum_{b \in B} b E$. If the conclusion of 2.5 is false then we can choose a relation $\sum_{i=1}^{r} t_{i} b_{i} e_{i}=0$ with $r$ minimal, where the $\left(t_{i}, b_{i}\right)$ are distinct elements of $T \times B$ and the $e_{i} \in E^{*}$. By multiplying on the right by $e_{1}^{-1}$ we may assume that $e_{1}=1$. Since $t_{1}$ is a unit and $b_{1} \neq 0$ we have $r>1$. 
Let $x \in E^{*}$. Then

$$
\begin{aligned}
0 & =t_{1}\left(t_{1}^{-1} \sum t_{i} b_{i} e_{i}\right)^{x}-\sum t_{i} b_{i} e_{i} \\
& =\sum_{i=2}^{r} t_{i} b_{i}\left(\left[t_{1}^{-1} t_{i}, x\right] e_{i}^{x}-e_{i}\right)
\end{aligned}
$$

where we have used that $\left[G, E^{*}\right] \leqq E^{*}$ commutes with $B$.

By the minimal choice of $r$ we have that $\left[t_{1}^{-1} t_{i}, x\right] e_{i}^{x}=e_{i}$ for all $x \in E^{*}$ and all $i>1$. Then $\left(t_{1}^{-1} t_{i} e_{i}\right)^{x}=t_{1}^{-1} t_{i} e_{i}$ for all $x \in E^{*}$ and $t_{1}^{-1} t_{i} e_{i} \in C$. Hence $t_{i} \in t_{1} C E$ and this is for all $i>1$. Thus $t_{i}=t_{1}$ and so $e_{i} \in C \cap E=F$ for all $i>1$. But we now have that $\sum b_{i} e_{i}=0$ and this contradicts the choice of $B$. The proof is complete.

\section{Hypercentral and hyperabelian groups}

3.1. Let $G$ be a locally nilpotent group and suppose that $G$ is a finite extension of a centre by periodic group. Then $G$ is centre by periodic.

Proof. By hypothesis there are subgroups $A \leqq C$ of $G$ with $A$ central in $C, C / A$ periodic and $C$ normal in $G$ with $G / C$ finite. We can replace $A$ by one of its free abelian subgroups of maximal rank. Now $A$ has at most $(G: C)$ conjugates in $G$ and therefore $A_{G}=\bigcap_{g \in G} A^{g}$ is normal in $G$ with $G / A_{G}$ periodic. Thus we may also choose $A$ normal in G.

Since $G$ is locally nilpotent it has a periodic normal subgroup $T$ with $G / T$ torsionfree. Clearly $G / T$ is the isolator of $A T T$ in $G / T$. Since centralizers in $G / T$ are isolated ([3] 4.8) it follows that $G / T$ is abelian. Consequently,

$$
[A, G] \leqq A \cap T=\langle 1\rangle
$$

since $A$ is torsion-free. That is $A$ lies in the centre of $G$.

3.2. Let $H$ be a normal subgroup of the absolutely irreducible subgroup $G$ of $G L(n, D)$ such that $H$ is hypercentral. Then $H$ is centre by locally-finite.

Proof. In view of $2.2,2.4$ and 3.1 we may assume that $G$ is prime. Set

$$
N=\Delta_{G}(H)=\left\{g \in G:\left|g^{H}\right|<\infty\right\}
$$

and $K=[G, H] \leqq H$.

Now $\Delta(H)$ is locally nilpotent and so is locally finite by torsion-free. It is also an $F C$ group and so is centre by periodic. Hence by [7] 4.3.2 the kernel $a$ of the natural map of the group algebra $F G$ onto $F[G]$ is annihilator free in $F G$ over $\Delta(H)$. Consequently a $\cap F N$ is annihilator free in $F N$ over $K \cap N \leqq \Delta(H)$. But then a is annihilator free in $F G$ over $K$ by [7] 3.2 and $F[G]$ is a crossed product of $F[N]$ by $G / N$. Consequently $G / N$ is periodic ([8] 2.2). 
We now have that $H \cap N=\Delta(H)$ is an $F C$-group and $H / \Delta(H)$ is periodic. Let $A$ be the centre of $\Delta(H)$ and set $Z=C_{A}(H)$. Clearly $A$ is normal in $H$ and $H / A$ is periodic. In particular ([3] 4.8 again) $H^{\prime}$ lies in the torsion subgroup of $H$. Let $a \in A$. Then $\left\langle a^{H}\right\rangle$ is finitely generated abelian and its torsion subgroup has finite order $m$ say. Hence $\left\langle a^{H}\right\rangle^{m}$ is torsion-free and normal in $H$, so $\left[\left\langle a^{H}\right\rangle^{m}, H\right] \leqq\left\langle a^{H}\right\rangle^{m} \cap T=\langle 1\rangle$. It follows that $a^{m} \in Z$ and consequently $A / Z$ is periodic. The result follows.

3.3 Corollary. Let $H$ and $G$ be as in 3.2. Then $G / G_{G}(H)$ is periodic.

This is an immediate consequence of 3.2 and [9] Theorem A(ii). The discerning reader will have seen that we have apparently not used the full force of the hypercentrality of $H$. We used in fact that $H$ is hyper $F C$-central. The following result shows in particular that this is not the case.

3.4. Let $G$ be a locally nilpotent group, unipotent-free when skew linear.

(a) If $G$ is skew linear then the torsion subgroup $T$ of $G$ lies in the hypercentre of $G$.

(b) $G$ is hypercentral if either $G$ is hyper FC-central or $G$ is skew linear and abelian by periodic.

Proof. (a) Since $G$ is skew linear $T$ satisfies Min- $p$ for every prime $p$. It follows that $T$ has an ascending series of normal subgroups of $G$ with finite factors. Each of these finite factors is $G$-hypercentral, so $T$ is too.

(b) Suppose $G$ is hyper $F C$-central. If $a \in \Delta_{T}(G)$ then $\left\langle a^{G}\right\rangle$ is finite. Thus again $T$ has an ascending series of normal subgroups of $G$ with finite factors and $T$ is $G$ hypercentral. Hence we may assume that $T=\langle 1\rangle$, and then centralizers in $G$ are isolated ([3] 4.8). Thus $\Delta(G)$ is the centre of $G$ and $G / \Delta(G)$ is torsion-free. Clearly the union of an ascending series of isolated subgroups of $G$ is isolated. An elementary transfinite induction completes the proof.

Suppose now that $G$ is skew linear and abelian by periodic. By [3] 4.8 again $G / T$ is abelian. Thus the result follows from part (a).

3.5. Let $H$ be a locally nilpotent normal subgroup of the absolutely irreducible subgroup $G$ of $G L(n, D)$ and suppose that $H \in P(\mathfrak{U} \cup L \mathfrak{F})$. Then $H$ is centre by periodic and $G / C_{G}(H)$ is periodic.

Proof. Now $H$ is abelian by periodic by [9] Theorem $\mathrm{A}(\mathrm{i})$. Hence $H$ is hypercentral by 3.4. Consequently $H$ is centre by periodic by 3.2 and $G / C_{G}(H)$ is periodic by 3.3.

Note that 1.1 is an immediate consequence of $3.2,3.3$ and 3.5 . Also 1.3 is an immediate consequence of 3.3 and 1.A.8 of [4]. By generalizing the latter result, which we now proceed to do, we can prove a little more.

3.6. Let $\mathfrak{X}$ be an $\left\langle N, P, Q, S_{n}\right\rangle$-closed class of groups. Let $H$ be a normal subgroup of a group $G$ such that $H$ has an ascending series of normal subgroups of $G$ with factors in 
A $\cup \mathfrak{X}$. Then $H$ contains normal subgroups $A \leqq T \leqq B$ of $G$ with $A=C_{H}(B), T / A \in \mathfrak{X}$ and $B / T \in \mathcal{A}$.

In the only application that we make of 3.6 we take $\mathfrak{X}$ to be the class of locally finite groups.

Proof. Let $A$ be maximal among the abelian normal subgroups of $G$ contained in $H$ (possibly $A=\langle 1\rangle$ ) and set $C=C_{H}(A)$. Let $T / A$ be the unique maximal normal $\mathfrak{X}$ subgroup of $C / A$; this exists since $N \mathfrak{X}=\mathfrak{X}$. Trivially $T$ is normal in $G$. If $T=C$ set $B=T$. Then $C_{H}(B) \leqq C_{H}(A)=B$ and the maximality of $A$ yields that $A=C_{H}(B)$.

Now assume that $T \neq C$. Since $\mathfrak{X}$ is $P$-closed, the choice of $T$ ensures that there is no non-trivial normal $\mathfrak{X}$-subgroup of $C / T$, and since $\mathfrak{X}$ is $Q$-closed $H / T$ has an ascending $G$ normal series with factors in $\mathfrak{A} \cup \mathfrak{X}$. Hence the $S_{n}$-closure of $\mathfrak{X}$ ensures the existence of a non-trivial abelian normal subgroup of $G / T$ in $C / T$, and we choose $B / T$ to be maximal among such. The maximal choice of $A$ yields that $A=B \cap C_{H}(B)$. It remains only to show that $C_{H}(B) \leqq B$, and clearly $C_{H}(B) \leqq C$.

Suppose $C_{H}(B) \leqq B$, so $A<C_{H}(B)$. Then there is a non-trivial normal subgroup $K / A$ of $G / A$ in $C_{H}(B) / A$ with $K / A$ in either $\mathfrak{U}$ or $\mathfrak{X}$. Since $A=B \cap C_{H}(B)$ we have $K \leq B$. If $K / A$ is abelian, then so is $B K / T$ and the maximal choice of $B$ would yield $K \leqq B$. If $K / A$ is an $\mathfrak{X}$-group the maximal choice of $T$ would yield $K \leqq T \leqq B$. These contradictions complete the proof.

3.7. Let $H$ be a normal subgroup of the absolutely irreducible skew linear group $G$ and suppose that $H$ has an ascending series of normal subgroups of $G$ with factors in $P(\mathfrak{H} \cup L \mathfrak{F})$. Then $H$ and $G / C_{G}(H)$ are abelian by periodic.

Proof. Any $P(\mathfrak{U} \cup L \mathfrak{F})$-group has a characteristic series of finite length with factors in $\mathfrak{A} \cup L \mathfrak{F}$ by [9] 3.4. Hence we can apply 3.6 and find a normal $P(\mathfrak{A} \cup L \mathfrak{F})$-subgroup $B$ of $G$ with $C_{H}(B) \leqq B \leqq H$. By [9] Theorem $A$ the factor $G / C_{G}(B)$ is abelian by periodic. Thus $H$ is metabelian by periodic and the same theorem, but applied now to $H$, yields that $H$ and $G / C_{G}(H)$ are abelian by periodic.

If we choose $H=G$ in 3.7 we obtain the following generalization of 1.3 .

3.8. Corollary. Let $G$ be an absolutely irreducible skew linear group such that $G \in \dot{P}_{n} P(\mathcal{H} \cup L \mathfrak{F})$. Then $G$ is abelian by locally-finite.

\section{Locally nilpotent groups and rings of quotients}

4.1. Let $R=S[G]$ be a ring, where $G$ is a locally nilpotent subgroup of the group of units of $R$ and $S$ is a subring of $R$ normalized by $G$. Suppose that every nilsubring of $R$ is nilpotent. For any subgroup $H$ of $G$ denote the nilpotent radical of $S[H]$ by $\mathrm{n}[H)$. Then

$$
\mathrm{n}(G)=\bigcup_{X} \mathrm{n}(X)=\bigcup_{H} \mathrm{n}(H)
$$

where $X$ ranges over the finitely generated subgroups of $G$ and $H$ over all subgroups of $G$. 
Proof. If $x \in \mathfrak{n}(G)$ there is a finitely generated subgroup $X$ of $G$ with $x \in S[X]$. Then $x \in S[X] \cap \mathfrak{n}(G) \subseteq \mathfrak{n}(X)$ and so

$$
n(G) \subseteq \bigcup_{X} \mathrm{n}(X), \quad=N \text { say. }
$$

Let $X$ and $Y$ be finitely generated subgroups of $G$ and set $Z=\langle X, Y\rangle$. Then $Z$ is nilpotent and there is a series $X=X_{0} \triangleleft X_{1} \triangleleft \cdots \triangleleft X_{r}=Z$ of finite length. Now $\mathrm{n}(X)$ is nilpotent and normalized by $X_{1}$, so $n(X) X_{1}$ is a nilpotent ideal of $S\left[X_{1}\right]$. Thus $\mathrm{n}(X) \subseteq \mathrm{n}\left(X_{1}\right)$. An elementary induction shows that $\mathrm{n}(X) \cup \mathrm{n}(Y) \subseteq \mathrm{n}(Z)$. It follows that $N$ is a locally nilpotent ideal of $S[G]$ and therefore $N \subseteq \mathrm{n}(G), \mathrm{n}(G)=\bigcup_{X}(X)$ and then

$$
\bigcup_{H} \mathrm{n}(H)=\bigcup_{H} \bigcup_{X \leqq H, X \in \mathfrak{G}} \mathrm{n}(X)=N=\mathrm{n}(G)
$$

as claimed.

4.2. Assume the notation of 4.1. If in addition $R$ is semiprime then $S[H]$ is semiprime for every subgroup $H$ of $G$.

For $S[H]$ is semiprime if and only if $n(H)=\{0\}$. Thus 4.2 follows from 4.1.

4.3. Corollary. Let $H$ be a locally nilpotent normal subgroup of the completely reducible subgroup $G$ of $G L(n, D)$ and let $S$ be a subring of $D^{n \times n}$ normalized by $G$. Suppose that $G$ is irreducible or $S=F$. Then the subring $S[K]$ of $D^{n \times n}$ is semiprime for every subgroup $K$ of $H$.

For any nilsubring of a simple Artinian ring is nilpotent by [1] 1.35 . Thus the irreducible case of 4.3 follows from 4.2 and 2.1. The case $S=F$ follows from applying the previous case to the irreducible constituents of $G$.

There is no need in 4.3 for $S[H]$ to be a domain, even if $S=F$ and $G$ is irreducible; for example $D=F, n=2, H$ the $2 \times 2$ diagonal group over $F$ and

$$
G=\left\langle H,\left(\begin{array}{ll}
0 & 1 \\
1 & 0
\end{array}\right)\right\rangle
$$

then $F[H] \cong F \oplus F$. An important step in Section 5 in the reduction to the case where $F[H]$ is a domain. (In fact $F[H]$ need not be a semidomain either.)

4.4. Let $G$ be an irreducible subgroup of $G L(n, D)$ and let $S$ be a right Ore subring of $D^{n \times n}$ normalized by $G$. Then the ring $Q$ of right quotients of $S$ is naturally embedded in $D^{n \times n}$.

If $G$ is reducible (e.g. $G=\langle 1\rangle$ ) this is false, even if $D$ is a field.

Proof. Let $V=D^{n}$ be row space, regarding as a $D-D^{n \times n}$ bimodule in the obvious way and let $C=\mathscr{C}_{S}(0)$, the set of regular elements of $S$. Since $S$ is right Ore the $C$-torsion subset $Z=\{v \in V: v c=0$ for some $c \in C\}$ is a $D-S$ submodule of $V$. But $G$ normalizes $C$, so 
in fact $Z$ is a $D-G$ submodule of $V$. By hypothesis $V$ is a $D-G$ irreducible, so $Z=V$ or $Z=\{0\}$.

If $Z=V$ then again by the Ore property there exists $c \in C$ killing a left $D$-basis of $V$. But then $V c=\{0\}$ and yet $c \neq 0$. Therefore $Z=\{0\}$ and each element of $C$ is injective on $V$. It follows that $C \subseteq G L(n, D)$. Clearly the subring $S\left[C^{-1}\right]$ of $D^{n \times n}$ is $S$-isomorphic to $Q$.

4.5. Let $S$ be semiprime right Ore subring of $D^{n \times n}$. Then for some integer $m \leqq n$ the ring $Q$ of right quotients of $S$ is isomorphic to a subring of $D^{m \times m}$. If also $S$ is right Goldie then $\operatorname{dim} S_{S} \leqq n$.

The dimension here is the uniform or Goldie dimension of $S$ as right $S$-module. The result is a generalization from prime to semiprime of an unpublished lemma of J. C. Robson and J. T. Stafford. In 4.5 we cannot necessarily extend the given embedding of $S$ in $D^{n \times n}$ to one of $Q$, even if $Q$ is in fact isomorphic to a subring of $D^{n \times n}$. For example let $D=E(x)$ where $E$ is any field and $x$ is an indeterminate, let $X=\operatorname{diag}(x, o)$ and set $S=E[X] \leqq D^{2 \times 2}$. Clearly $S$ projects onto $E[x]$ via the $(1,1)$ entries, so $S \cong E[x]$ is a domain and $Q$ exists and is isomorphic to $D$. But $X$ is clearly a zero-divisor of $D^{2 \times 2}$ so the given embedding of $S$ in $D^{2 \times 2}$ does not extend to $Q$.

Proof. Regard $V=D^{n}$ as a $D-S$ bimodule in the usual way and let $V_{1}, V_{2}, \ldots, V_{r}$ be a full set of non-isomorphic $D-S$ composition factors of $V$. Let $p_{i}$ be the annihilator of $V_{i}$ in $S$. If $\mathfrak{a}$ is an ideal of $S$ then $V_{i} \mathfrak{a}$ is a $D-S$ submodule of $V_{i}$, which is irreducible. It follows that each $\mathfrak{p}_{i}$ is prime. Also $\bigcap \mathfrak{p}_{i}$ acts nilpotently on $V$ and $S$ is semiprime and faithful on $V$. Therefore $\bigcap p_{i}=\{0\}$. It follows that every prime ideal contains at least one of the $\mathfrak{p}_{i}$. Suppose $\mathfrak{p}_{1} \ldots, \mathfrak{p}_{s}$ are distinct and are all the minimal prime ideals of $S$. Then $\bigcap_{i=1}^{s} \mathfrak{p}_{i}=\{0\}$ and we have ring embeddings

$$
S \hookrightarrow \bigoplus_{i \leqq s} S / \mathfrak{p}_{i} \rightarrow \bigoplus_{i \leqq s} \operatorname{End}_{D} V_{i} \hookrightarrow \operatorname{End}_{D}\left(\bigoplus_{i \leqq s} V_{i}\right) \cong D^{m \times m}
$$

where $m=\sum_{i \leqq s} \operatorname{dim}_{D} V_{i} \leqq n$.

Let $C=\mathscr{C}_{S}(0)$ be the set of regular elements of $S$; by hypothesis $C$ is a right divisor set in $S$. Trivially $C_{i}=C+\mathfrak{p}_{i} / \mathfrak{p}_{i}$ is a multiplicative submonoid of $S_{i}=S / \mathfrak{p}_{i}$ and $S_{i}$ is right Ore with respect to $C_{i}$. Let $i \leqq s$. Then $\mathfrak{p}_{i}=l_{S} r_{s}\left(\mathfrak{p}_{i}\right)$ since $D^{n \times n}$ and hence $S$ satisfies the maximal condition on annihilators ([1] 1.16). Let $c \in C$ and $a \in S$ and suppose that $c a \in \mathfrak{p}_{i}$. Then $c a \cdot r_{S}\left(\mathfrak{p}_{i}\right)=\{0\}$ and yet $c \in \mathscr{C}_{S}(0)$. Thus $a \cdot r_{S}\left(\mathfrak{p}_{i}\right)=\{0\}$ and so $a \in l_{S} r_{s}\left(p_{i}\right)=p_{i}$. Also $\mathfrak{p}_{i}=r_{s} l_{s}\left(\mathfrak{p}_{i}\right)$ and the same argument shows that if $a c \in \mathfrak{p}_{i}$ then $a \in \mathfrak{p}_{i}$. This proves that $C_{i}$ is a set of regular elements of $S_{i}$ and so $C_{i}$ is a right divisor set in $S_{i}$. Thus we can form the ring $S_{i} C_{i}^{-1}$ of right quotients. Clearly the natural embedding of $S$ into $\bigoplus_{i \leqq s} S_{i}$ extends to one of $Q=S C^{-1}$ into $\bigoplus_{i \leqq s} S_{i} C_{i}^{-1}$.

By definition $V_{i}$ is an $S_{i}$-faithful $D-S_{i}$ bimodule. Let

$$
Z_{i}=\left\{v \in V_{i}: v c=0 \text { for some } c \in C_{i}\right\}
$$

be the $C_{i}$-torsion submodule of $V$. If $Z_{i}=V_{i}$ then some element of $C_{i}$ kills a left $D$-basis of $V_{i}$ and so kills $V_{i}$. This impossibility shows that $Z_{i}=\{0\}$ and so $C_{i}$ is a set of $D$. 
automorphisms of $V_{i}$. Therefore the natural embedding of $S_{i}$ in $\operatorname{End}_{D} V_{i}$ extends to one of $S_{i} C_{i}^{-1}$ and we have ring embeddings

$$
Q \hookrightarrow \underset{i \leqq s}{\bigoplus} S_{i} C_{i}^{-1} \hookrightarrow \underset{i \leqq s}{\bigoplus} \operatorname{End}_{D} V_{i} \hookrightarrow D^{m \times m}
$$

Always $\operatorname{dim} S_{S}=\operatorname{dim} Q_{Q}$. Suppose $S$ is right Goldie. Then $Q$ is semisimple Artinian, so $\operatorname{dim} Q_{Q}$ is the maximal number of orthogonal idempotents in $Q$. The maximal number of orthogonal idempotents in $D^{m \times m}$ is $m$. Therefore

$$
\operatorname{dim} S_{S}=\operatorname{dim} Q_{Q} \leqq m \leqq n
$$

\subsection{Proof of 1.2 .}

By 4.3 the ring $S[K]$ is semiprime for every subgroup $K$ of $H$. Trivially $S[K]$ has the maximal condition on right annihilators. Suppose $\bigoplus_{i=1}^{m} U_{i} \subseteq S[K]$, where the $U_{i}$ are non-zero right ideals of $S[K]$. There exists a finitely generated subgroup $X$ of $K$ such that each $U_{i} \cap S[X] \neq\{0\}$. Now $S[X]$ is right Noetherian by a theorem of P. Hall ([5] 10.2.7) and hence $S[X]$ is right Goldie and, as remarked above, semiprime. Hence $m \leqq \operatorname{dim} S[X]_{S[X]}$, $\leqq n$ by 4.5 . It follows that $\operatorname{dim} S[K]_{S[K]} \leqq n$ and consequently $S[K]$ is right Goldie.

\section{The structure of locally nilpotent groups}

Let $H$ be a locally nilpotent normal subgroup of the absolutely irreducible subgroup $G$ of $G L(n, D)$. In this section we show how to break $H$ and $G$ into a number of pieces. Although this is not strong enough to decide whether $H$ is centre by periodic, it does give quite a good description of how $G$ and $H$ are built up. Our notation in this section is accumulative.

Set $R=F[G]=D^{n \times n}$ and $S=F[H]$. By 2.2 there is a normal subgroup $G_{0}$ of $G$ of finite index and persistent groups $G_{1}, G_{2}, \ldots, G_{r}$ such that $G_{0}$ is a subdirect product of the $G_{i}$. Let $H_{i}$ be the natural image of $H \cap G_{0}$ in $G_{i}$. Then $H$ is a finite extension of a subgroup of the direct product of the $H_{i}$. In particular $H$ is centre by periodic whenever each $H_{i}$ is centre by periodic by 3.1. Thus in view of 2.4 we make the following extra hypothesis.

\subsection{Assume that $G$ is prime.}

Thus $S$ is now prime as well as Goldie by 1.2. Then by 4.4 the ring $Q$ of quotients of $S$ is embedded naturally in $R$ and is simple Artinian ([1] 1.28). Thus $Q=E U$ for some division ring $E$ and set $U$ of matrix units centralizing $E$. There exists a regular element $s$ of $S$ with $U s \subseteq S$. Let $Y$ be any finitely generated subgroup of $H$ with $s \in F[Y]$ and $U s \subseteq F[Y]$.

$F[Y]$ is semiprime and Goldie by 1.2 (in fact it is even Noetherian). Let $Q_{Y}$ be its ring of quotients. Since $U S \subseteq F[Y]$ it follows that $Y$ is an irreducible subgroup of the group of units of $Q$. Hence we can choose $Q_{Y}$ in $Q$ by 4.4 again. Now $s$ is regular in $F[Y]$ since it is in $S$, so $s^{-1} \in Q_{Y}$. Thus $U \subseteq Q_{Y}$ and $Q_{Y}$ is a matrix ring over $E \cap Q_{Y}$ with $U$ as 
a set of matrix units ([5] 6.1.5). But $E \cap Q_{Y}$ is a domain, so $Q_{Y}$ is a prime and yet $Q_{Y}$ is semisimple Artinian ([1] 1.27). Consequently $Q_{Y}$ is simple and $F[Y]$ is prime by [1] 1.28 .

Let $T$ denote the torsion subgroup of $H$ and $Z / T$ the centre of $H / T$. Then 2.5 yields the following.

5.2. $F[H]$ is a crossed product of $F[Z]$ by the torsion-free locally nilpotent group $H / Z$.

The torsion-freeness of $H / Z$ is yet another application of [3] 4.8(a). Set $K=C_{H}(Z)$ and $A=K \cap Z$.

5.3. $H / K$ is periodic. $H$ is centre by periodic if (and trivially only if) $K$ is centre by periodic.

For $G / C_{G}(Z)$ is periodic by 1.1. Thus $H / K$ is periodic. The second part of 5.3 then also follows from 1.1 .

\section{4. $A$ is the centre of $K$.}

Trivially $A$ lies in the centre of $K$. By [3] 4.8(a) the centralizer of the centre of $K T / T$ in $H / T$ is isolated and hence is $H / T$. Consequently the centre of $K T / T$ is $(K T \cap Z) / T=$ $A T / T$ and the centre of $K$ lies in

$$
A T \cap K=A(T \cap K) \subseteq A(Z \cap K)=A
$$

By 5.2 we have the following.

5.5. $F[K]$ is a crossed product of $F[A]$ by the torsion-free locally nilpotent group $K / A$.

But $F[A]$ is a domain by 5.1 , so $F[K]$ is also a domain by Higman's zerodivisor theorem. It is also Ore by 1.2 and therefore the ring $L$ of quotients of $F[K]$ is a division ring. By 4.4 the ring $L$ is embedded naturally in $R$, and hence in $Q$. Set

$$
X=G \cap L^{*} C_{L^{*} G}(K)=G \cap N_{L^{*}}(K) C_{L^{*} G}(K) \supseteq K .
$$

By 2.6 applied with $L$ and $L^{*} G$ for $E$ and $G$ the ring $R$ is a crossed product of $L\left[C_{R}(K)\right]$ by $G / X$. But $R=F[G]$, so using [8] 2.2 to obtain the periodicity of $G / X$ we have the following.

5.6. $R$ is a crossed product of $F[X]=L\left[C_{R}(K)\right] \supseteq K C_{G}(K)$ by the periodic group $G / X$.

Let $C$ be the centre of $L$. Since $L$ is a central simple $C$-algebra, $F[X]=L\left[C_{R}(K)\right]$ is the tensor product over $C$ of $L$ and $C_{R}(K)$. But $F[X]$ by the definition of $X$ is generated by $C\left[N_{L^{*}}(K)\right]$ and $C_{R}(K)$. Therefore

5.7. $L=C\left[N_{L^{*}}(K)\right]$. Also $K \cap C=A$. 
We have now completed our analysis of $G$ and $H$. Notice that $\left(L, C, N_{L^{*}}(K), K\right)$ in place of $(D, F, G, H)$ satisfies Condition $\left({ }^{*}\right)$ of Section 1. If in the special case of $\left(^{*}\right)$ the group $H$ is centre by periodic, then the group $K$ above is also centre by periodic and consequently in general $H$ is too by 5.3 .

\section{Radical groups}

6.1. Proof of 1.4 .

We are assuming here that $H \in \dot{P} L(\mathfrak{N} \cup \mathfrak{F})$. If $X \in L(\mathfrak{N} \cup \mathfrak{F})$ is not locally nilpotent, it is easily seen that $X$ is locally finite. Thus by the Hirsch-Plotkin theorem

$$
\dot{P} L(\mathfrak{N} \cup \mathfrak{F})=\dot{P}_{n}(L \mathfrak{R} \cup L \mathfrak{F})
$$

Let $K$ be the Hirsch-Plotkin radical of $H$ and let $T / K$ be the maximal locally finite normal subgroup of $H / K$. By hypothesis $K$ is centre by periodic, so the group $G / C_{G}(T)$ is abelian by periodic. Let $C$ be the centre of $T$. If $C=C_{H}(T)$ then $H$ is abelian by periodic and Theorem $A$ of [9] shows that $G / C_{G}(H)$ is too.

Suppose $C \neq C_{H}(T)$. Then there is a non-trivial normal subgroup $L / C$. of $H / C$ in $C_{H}(T) / C$ that is either locally nilpotent or locally finite. If $L / C$ is locally nilpotent then so is $L$ and $L \subseteq K \cap C_{H}(T) \subseteq C$. If $L / C$ is locally finite then $C \leqq K$ and $L \leqq T \cap C_{H}(T)=C$. Thus neither case is possible and the proof is complete.

6.2. Remark. Free groups lie in both $\grave{P}_{n} \mathfrak{A}$ and $\grave{P}_{n} \mathfrak{F}$. Thus there is no hope of comparable results if one works with series that are not ascending. It would be interesting if one could say something about the class of locally soluble skew linear groups.

\section{REFERENCES}

1. A. W. Chatters and C. R. Hajarnavis, Rings with Chain Conditions (Pitman, London etc. 1980).

2. E. Formanek and A. V. Jategaonkar, Subrings of Noetherian rings, Proc. Amer. Math. Soc. 46 (1984), 181-186.

3. P. Hall, The Edmonton Notes on Nilpotent Groups (Queen Mary College Math. Notes 1969).

4. O. H. Kegel and B. A. F. Wehrfritz, Locally Finite Groups (North-Holland Pub. Co., Amsterdam etc. 1973).

5. D. S. Passman, The Algebraic Structure of Group Rings (John Wiley \& Sons, New York etc. 1977).

6. D. J. S. Robinson, Finiteness Conditions and Generalized Soluble Groups Vol. 1 (SpringerVerlag, Berlin etc. 1972).

7. B. A. F. Wenrfritz, Soluble by periodic skew linear groups, Math. Proc. Cambridge Philos. Soc. 90 (1984), 379-389.

8. B. A. F. Wehrfritz, Locally finite normal subgroups of absolutely irreducible skew linear groups, J. London Math. Soc. (2) 32 (1985), 88-102. 
9. B. A. F. Wehrfritz, Soluble normal subgroups of skew linear groups. J. Pure \& Appl. Alg., to appear.

10. B. A. F. Wehrfritz, On absolutely irreducible skew linear groups in characteristic zero, Arch. Math. (Basel), 45 (1985), 193-199.

11. A. E. Zalesskir, The structure of several classes of matrix groups over a division ring (Russian, Sibirsk. Mat. Ž ; 8 (1967), 1284-1298 = Siberian Math. J. 8 (1967), 978-988).

Queen Mary College

LONDON E1 4NS 